\title{
Pacing strategy of a wheelchair athlete in a 5x and 10x Ironman ultra triathlon: a case study
}

Sousa, Caio Victor ; Nikolaidis, Pantelis Theodoros ; Andrade, Marilia Santos ; Vancini, Rodrigo Luiz ; Barbosa de Lira, Claudio Andre ; Puccinelli, Paulo José Gomes ; Knechtle, Beat

\begin{abstract}
Objective: For disabled athletes such as wheelchair athletes, there is no knowledge about competing and pacing during a long-distance triathlon such as an Ironman triathlon. This study aimed to investigate the pacing strategy of a paraplegic wheelchair athlete competing and finishing a Quintuple Iron ultra-triathlon (i.e., five times $3.8 \mathrm{~km}$ swimming, $180 \mathrm{~km}$ handbike cycling and $42.195 \mathrm{~km}$ wheelchair racing in five days) and a Deca Iron ultra-triathlon (10 times the same distance in 10 days). Methods: Data from an ultra-distance triathlon race (Swissultra) covering 5x and 10x Ironman distance were collected. Official performance data were acquired from the race organizer's website and athlete's personal information from the athlete through online interviews. The athlete is a man born in 1962, the races analysed in this study were held in the summer of 2017 (5x) and 2019 (10x). The split times for swimming, cycling and running, the overall race times for each Ironman and the lap times in cycling (handbike) and running (wheelchair) were analysed. Results: The athlete finished the Quintuple Iron ultra-triathlon in an overall race time of 66:28:31 h:min:s and the Deca Iron ultra-triathlon in 137:03:20 h:min:s. He adopted an even pacing in both races in split disciplines and for overall race time. Conclusion: The paraplegic wheelchair athlete was able to finish both a Quintuple and a Deca Iron ultra-triathlon by adopting an even pacing in all split disciplines and for overall race time. IMPLICATIONS FOR REHABILITATION Triathlon is a growing sport among athletes with spinal cord injury. Ultra-triathlons are ultra-endurance events and pacing is a key aspect to a successful race regardless the athlete's category. An athlete with a spinal cord injury finished a $5 \mathrm{x}$ and 10x Ironman ultra-triathlon applying an even pacing strategy.
\end{abstract}

DOI: https://doi.org/10.1080/17483107.2020.1807620

Posted at the Zurich Open Repository and Archive, University of Zurich

ZORA URL: https://doi.org/10.5167/uzh-189911

Journal Article

Accepted Version

Originally published at:

Sousa, Caio Victor; Nikolaidis, Pantelis Theodoros; Andrade, Marilia Santos; Vancini, Rodrigo Luiz; Barbosa de Lira, Claudio Andre; Puccinelli, Paulo José Gomes; Knechtle, Beat (2022). Pacing strategy of a wheelchair athlete in a $5 \mathrm{x}$ and 10x Ironman ultra triathlon: a case study. Disability and Rehabilitation. Assistive Technology, 17(6):668-674.

DOI: https://doi.org/10.1080/17483107.2020.1807620 
1 Pacing Strategy of a Wheelchair Athlete in a 5x and 10x Ironman Ultra Triathlon: a

2 case study

3 Running head: Ultra Triathlon Pacing in a Wheelchair

4

\section{Abstract}

6 Objective: For disabled athletes such as wheelchair athletes, there is no knowledge about 7 competing and pacing during a long-distance triathlon such as an Ironman triathlon. This

8 study aimed to investigate the pacing strategy of a paraplegic wheelchair athlete competing

9 and finishing a Quintuple Iron ultra-triathlon (i.e. five times $3.8 \mathrm{~km}$ swimming, $180 \mathrm{~km}$

10 handbike cycling and $42.195 \mathrm{~km}$ wheelchair racing in five days) and a Deca Iron ultra-

11 triathlon (10 times the same distance in 10 days).

12 Methods: Data from an ultra-distance triathlon race (Swissultra) covering 5x and 10x

13 Ironman distance were collected. Official performance data were acquired from the race

14 organizer's website and athlete's personal information from the athlete through online

15 interviews. The athlete is a man born in 1962, the races analyzed in this study were held in

16 the summer of 2017 (5x) and 2019 (10x). The split times for swimming, cycling and running,

17 the overall race times for each Ironman and the lap times in cycling (handbike) and running

18 (wheelchair) were analyzed.

19 Results: The athlete finished the Quintuple Iron ultra-triathlon in an overall race time of

20 66:28:31 h:min:s and the Deca Iron ultra-triathlon in 137:03:20 h:min:s. He adopted an even

21 pacing in both races in split disciplines and for overall race time.

22 Conclusion: The paraplegic wheelchair athlete was able to finish both a Quintuple and a

23 Deca Iron ultra-triathlon by adopting an even pacing in all split disciplines and for overall

24 race time.

25 Key words: swimming, cycling, running, disabled athlete, spinal cord injury 
Implications for Rehabilitation

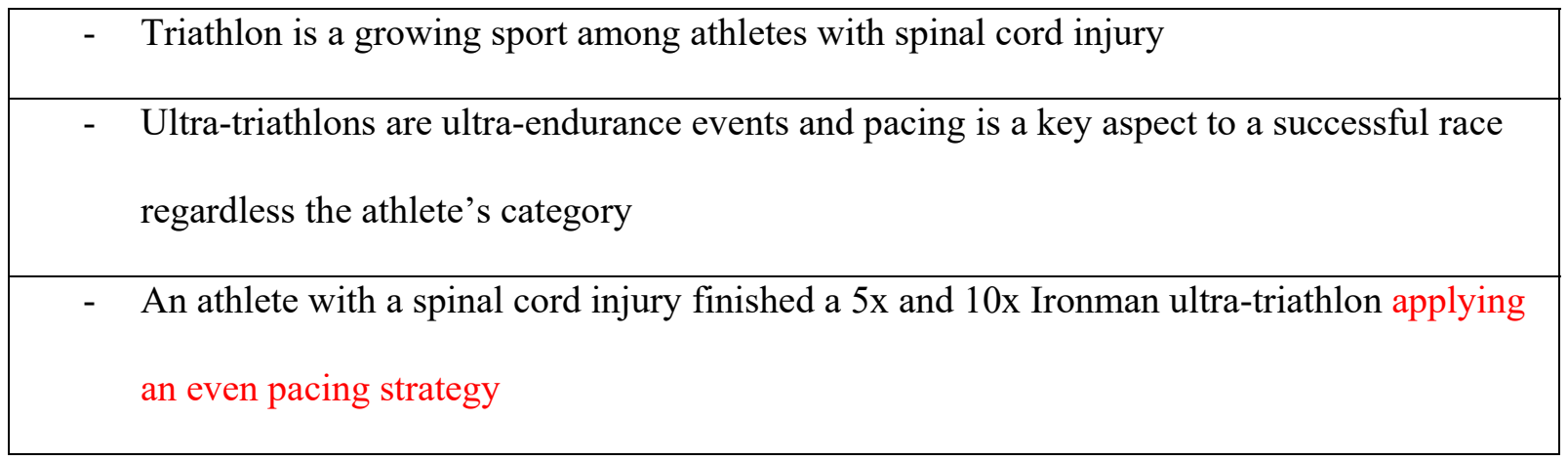

27

\section{Introduction}

30 Ironman triathlon is of high popularity where both the number of races and athletes in the last years

31 has dramatically increased [1]. Furthermore, the performance of elite [2,3] and recreational Ironman

32 triathletes [4] has improved in the last decades. Pacing is an important aspect in endurance

33 performance in general [5] and also in triathlon [6]. Regarding Ironman triathlon, pacing has been

34 investigated during the cycling and running split [7-9]. For both female and male elite Ironman

35 triathletes, a positive pacing (i.e. slowing down) for both cycling and running has been described [7].

37 The distribution of speed, work, or pacing throughout an exercise task is very important to optimize

38 performance $[10,11]$. Triathlon provides a unique model for pacing analysis due to the involvement

39 of sequential swimming, cycling and running [6], and a proper strategy is fundamental in ultra-

40 endurance triathlon [12]. Intrinsically, research shows that pacing is partially controlled on a

41 subconscious level [13] and partially by "pacing strategy" [6]. In a triathlon race, pacing is affected

42 by variety of external factors, such as sea currents, wind speed, topography $[14,15]$ or even

43 individual factors, such as gender or age [12]. Additional challenges that may affect pacing and

44 performance in ultra-endurance triathlons performed in more than one race day included sleep,

45 recovery and nutrition [16]. 
47 Disabled athletes such as wheelchair athletes perform sport primarily in basketball [17], rugby [18]

48 and wheelchair racing [19]. To date, no data exists about a wheelchair athlete competing in an

49 Ironman and no study has ever investigated how a wheelchair athlete would pace during an Ironman

50 triathlon. Compared to their able-bodied counterparts, wheelchair Ironman triathletes relied on

51 skeletal muscles of trunk and upper limbs [20]. In addition, upper and lower limbs showed different

52 patterns of total, central (e.g. greater in knee extensors) and peripheral fatigue (e.g. greater in elbow

53 flexors) [21].

54

55 In this case study, we describe the pacing of the first and only wheelchair athlete worldwide to

56 compete and finish a Quintuple Iron ultra-triathlon (i.e. 5 Ironman triathlons in 5 days) and a Deca

57 Iron ultra-triathlon (i.e. 10 Ironman triathlons in 10 days). Based upon existing findings in pacing

58 during a Deca Iron ultra-triathlon in able-bodied athletes [22,23], the hypothesis is a positive pacing

59 (i.e. slowing down over days) also in a disabled athlete during both a Quintuple and a Deca Iron

60 ultra-triathlon. 


\section{$61 \quad$ Materials and Methods}

\section{Ethical approval}

64 This study was approved by the Institutional Review Board of Kanton St. Gallen, Switzerland, with a 65 waiver of the requirement for informed consent of the participants as the study involved the analysis 66 of publicly available data.

67

\section{Races characteristics}

69 The Swissultra (www.swissultra.ch) is an ultra-distance triathlon held since 2016 where races

70 covering 5x, 10x or 20xIronman (i.e. $3.8 \mathrm{~km}$ swimming, $180 \mathrm{~km}$ cycling and $42.195 \mathrm{~km}$ running) are

71 held. Swimming is held in a 50 m outdoor pool with a temperature of $20-23{ }^{\circ} \mathrm{C}$. Cycling is held on a

72 completely flat and traffic-free course where 20 laps of $9 \mathrm{~km}$ must be performed. Running is held on

73 a completely free course where 35 laps of $1.2 \mathrm{~km}$ must be completed. Each lap is measured

74 electronically with a chip system.

75

76 The race is held in the last two weeks of August where temperatures vary during the day from 25-35

$77{ }^{\circ} \mathrm{C}$. Often, rain falls in the late afternoon and evening. The cycling course is held in a large and broad

78 valley (Rheintal) where the first half turns from north to south to a turning point to complete the

79 second half from south to north. Early in the morning the wind is blowing from south to north and

80 changes before noon from north to south.

81

82 Both Quintuple and Deca ultra-triathlon have an overall time limit of 20 hours per day and a specific

83 time limit for swimming (2 hours; started every day at 7:00 a.m.), cycling (up to 11:00 p.m.) and

84 running (3:00 a.m. of the following day). 
87 The athlete (57 years, $170 \mathrm{~cm}, 56 \mathrm{~kg}$ ) is born in 1962 and competed in 2017 at the age of 55 years in

88 the Quintuple Iron ultra-triathlon held in August in Buchs, Switzerland, and in 2019 at the age of 57

89 years in the Deca Iron ultra-triathlon. He suffers from a complete paraplegia L1 since 1998. He trains

90 for 15-20 hours per week. During summer, he trains primarily handbike cycling, but seldom

91 wheelchair racing. During winter, he trains cross-country skiing. Since several years he is a member

92 of the Swiss national team in handbike cycling and competes in handbike cycling races where he

93 won the European cup in marathon cycling and in 2010, he was the overall winner of the European

94 cup. He also competed in a team in the Gigathlon (www.gigathlon.com), a multi-day race consisting

95 of swimming, cycling, running, inline skating and mountain biking) held in Switzerland where he

96 took the part of cycling in a team. Apart from the Gigathlon, he competed in a team in the Tortour

97 (www.tortour.com), a cycling race around Switzerland.

99 Regarding handbike training, he completed between 2004 and 2010 about 12,000 to 14,000 km per

100 year, since 2011 he favors handbike cycling in the mountains with 8,000 to 9,000 km per year. The

101 athlete stated different routines depending for the winter, with only indoor 10-12 hours of weekly

102 training performed on a spinning bike. Outdoor training begins in April with the handbike, with

103 long-distance range up to $100 \mathrm{~km}$ per day and $500 \mathrm{~km}$ per week (20-25 hours/week). In May, the

104 focus on endurance is uphill, with approximately $300 \mathrm{~km}$ or 20-25 hours/week. June is characterized

105 by interval trainings, volume ranging 20-25 hours per week, and two or three races to test optimal

106 pacing strategies. July has a decreased intensity and volume, 15-20 hours/week, and is used as

107 tapering the physical fitness and test race equipment. The athlete's training regimen were not

108 designed by an exercise specialist or coach, the athlete made up with his own experience and decided 109 the specifics of each training session before it starts depending on the weather and self-perception of 110 fatigue/fitness. 
112 The handbike he used during the Swissultra was designed and built especially for him. It is a $113 \mathrm{knee} / \mathrm{seat}$ bike on three wheels. The seat takes $80 \%$ of the body weight, and the remaining $20 \%$ is

114 knee support area. The seat and crank height are adjusted to the athlete's height. The bicycle

115 components are analogous to the racing bikes [24]; the racing class of the athlete is H5 at UCI-

116 Cycling [25]. The wheelchair for the marathon split was a Top End racing wheelchair

117 (https://topendwheelchair.invacare.com/racing-chairs/category/racingchairs-topend).

\section{Race strategy of the athlete}

120 The athlete adopted for both races the same strategy. He lives in Schaan, Principality of

121 Liechtenstein, which is 5 minutes by car away from Buchs, Switzerland. He lives alone in an

122 apartment without a family.

123

124 During the races, a crew of 17 persons worked in shifts: crew member $\mathrm{A}$ - swimming 6:30 a.m. to

125 9:30 a.m.; crew member B and C - handbike route 9:00 a.m. to 3:00 p.m.; crew member D -

126 marathon 3:00 p.m. to the end; different crew the next day. The crew members were responsible for

127 his food, drinks, clothes, utensils and mechanics. The shifts were divided by the disciplines and

128 changed during the transitions from one discipline to the other. For each discipline (swimming,

129 handbike and wheelchair) they had to provide his specific equipment and clothes. When there was a

130 period of rain, he made a short break and the crew helped in changing clothes.

132 He slept at home and ate the first muesli after getting up. The swim started every morning at 7 a.m.

133 After swimming, he made a short break and ate a second muesli at around 9 a.m. A little later, he

134 took a sandwich as a snack. After $90 \mathrm{~km}$ on the handbike, he took a lunch break of 20 min at around

1351 p.m. and ate the first half of lunch offered by the organizer. After eating, he went to the toilet. He 
136 then did another lap of handbike, ate then the second half of the lunch and took coffee and cake. At 137 around 4 p.m., he took another full meal offered by the organizer with the intention that the food 138 should be digested by around 5:30 p.m. before the start of the split with the racing wheelchair.

139 During the marathon split with the racing wheelchair, he was mostly fed with small snacks such as

140 bananas, figs, pretzel sticks, biscuits by his support crew. He also drank a carbohydrate electrolyte

141 drink. Due to the fact that he wore special gloves for the wheelchair, all food and drinks were

142 provided bite-sized by his support crew. At 9:30 p.m. he ate potatoes and cheese. After each stage,

143 he drove alone back home by car. When he got home every evening after an Ironman, he had to

144 cook, take a shower, wash clothes, eat dinner, and relax. He was able to sleep in his own bed and was

145 well recovered in the morning for the next start.

147 The athlete had an initial pacing goal to finish within the time limit of the race maintaining an 148 optimal pacing strategy expecting small and linear performance decreases every race day.

150 Data analysis

151 Data were obtained from the official race website of Swissultra at www.swissultra.ch/de/resultate.

152 We collected the split times for swimming, cycling and running, the overall race times for each

153 Ironman and the lap times in cycling (handbike) and running (wheelchair).

155 Each discipline (swimming, cycling (handbike) and running (wheelchair)) was performed in small 156 loops, with time being recorded for every lap. Average speed and average pace were calculated for 157 each lap, as well as average performance for each discipline in each race day. Data from each day of 158 the Quintuple and Deca Iron ultra-triathlon were displayed in overall and split analyses. Data are 159 expressed as mean, standard deviation (SD) and the coefficient of variation (\%). The coefficient of 160 variation $(\mathrm{CV})$ is the a relative $(\%)$ measure of dispersion of the data, calculated as $C V=\sigma / \mu^{*} 100$ 
161 (standard deviation/average*100). A higher CV means a higher variation of the pace. Repeated

162 measures ANOVA with within- between-interactions was applied to detect interactions in average

163 performance throughout the days (within; time) for handbike and wheelchair (between; group).

164 ANOVA analysis had a power (1-beta) of $67 \%$ and $53 \%$ to detect a large effect size $(d \geq 0.7)$ in the

165 Quintuple and Deca races, respectively. Non-linear regressions (second and third order) were used in

166 selected variables to identify possible patterns throughout race days. Pearson's correlation coefficient

167 was applied to test the association between the variables. The significance level was set as $p<0.05$.

168 Statistical Software for the Social Sciences was used in all statistical procedures (SPSS v25,

169 Chicago, Ill, USA). 
172 Table 1 shows the split and overall race time in each day for the Deca Iron ultra-triathlon of the

173 athlete. The total race time was 137 hours 3 minutes and 20 seconds. Table 2 shows the split and

174 overall race time in each day for the Quintuple Iron ultra-triathlon. The total race time was 66 hours

17528 minutes and 31 seconds.

176

177 [Table 1 near here]

178

179 [Table 2 near here]

180

181 Figure 1 displays the average speed to complete each lap in handbike and wheelchair in each day in 182 the Deca Iron ultra-triathlon. The data show a repeatable down in the handbike split between 81 and 183108 (i.e. middle of the split) in almost every race day. For the wheelchair split (i.e. the marathon), no 184 pattern for a pacing variation can be visualized throughout the days.

\section{6 [Figure 1 near here]}

188 Figure 2 displays the average speed to complete each lap in both the handbike and the wheelchair 189 split in each day in the Quintuple Iron ultra-triathlon. Similar to the Deca Iron ultra-triathlon, the 190 data show a repeatable down in the handbike split between 81 and 108 (i.e. middle of the split) in 191 almost every race day. For the wheelchair split, no pattern for pacing variation can be visualized 192 throughout the days. 
196 The average speed in each day was always higher in the handbike compared to the wheelchair split $197(\mathrm{p}<0.05)$ and no time-effect was found to neither handbike or wheelchair in the Deca Iron ultra198 triathlon (Figure 3-A). Similarly, in the Quintuple Iron ultra-triathlon, the average speed in each day 199 was always higher in the handbike compared to the wheelchair split $(\mathrm{p}<0.05)$ and no time-effect was 200 found in neither handbike or wheelchair (Figure 3-B). An even pacing was found in both the Deca 201 and the Quintuple Iron ultra-triathlon in the swim split $\left(\mathrm{R}^{2}=0.75 ; \mathrm{R}^{2}=0.59\right.$, respectively), in the 202 handbike split $\left(R^{2}=0.41 ; R^{2}=0.91\right.$, respectively), in the wheelchair split $\left(R^{2}=0.29 ; R^{2}=0.62\right.$, 203 respectively) and for overall race time $\left(\mathrm{R}^{2}=0.23 ; \mathrm{R}^{2}=0.76\right.$, respectively).

$205 \quad$ [Figure 3 near here]

207 The coefficient of variation (CV) in Deca Iron was similar throughout the first six days for the 208 wheelchair, with the highest CV in day $7(18.9 \%)$ and the lowest in day $8(6.0 \%)$. The handbike 209 started (Day 1) with the highest CV, and had the lowest on the last day (8.3\%). In the Quintuple Iron 210 ultra-triathlon, the CV was very stable $(\sim 14 \%)$ for the handbike throughout the five race days. For

211 the wheelchair split, the CV started very high (17.6\%), became stable in the middle days and

212 increased again in the last day (14.2\%).A third-order polynomial equation was able to fit CV pattern 213 along the race for Quintuple Iron ultra-triathlon (handbike $\mathrm{R}^{2}=0.98$; wheelchair $\mathrm{R}^{2}=0.99$ ), but for 214 the Deca Iron ultra-triathlon only handbike fitted (handbike $\mathrm{R}^{2}=0.98$; wheelchair $\mathrm{R}^{2}=0.01$ )

215 (Figure 4).

\section{7 [Figure 4 near here]}


219 In the Deca Iron ultra-triathlon, the correlation analysis showed a negative correlation between

220 performance and $\mathrm{CV}$ for the handbike, but not for the wheelchair split. Conversely, a positive

221 correlation was identified between performance and CV for the handbike in the Quintuple Iron ultra-

222 triathlon, with a negative correlation between performance and CV for the wheelchair (Figure 5).

223

$224 \quad$ [Figure 5 near here]

225

226

227

228

229

230

231

232

233

234

235

236

237

238

239

240

241

242

243 
246 This case study investigated the pacing during the cycling (i.e. handbike) and running (i.e.

247 wheelchair) split in a disabled athlete competing in both a Quintuple and a Deca Iron ultra-triathlon.

248 Based upon previous reports about pacing in a multi-stage triathlon, it was assumed that the disabled 249 athlete would become slower across days as well as across split disciplines.

251 The results show, however, an even pacing [5] for swimming, the handbike split (i.e. cycling part), 252 the wheelchair split (i.e. running part) and for overall race time during both the Quintuple and the 253 Deca Iron ultra-triathlon. These findings are in contrast to elite Ironman triathletes where 254 performance decreased (i.e. positive pacing) during both the cycling and running split [7] and to 255 Deca Iron ultra-triathletes with a decrease in performance across days in both split disciplines and 256 overall race time $[22,23]$.

258 A potential explanation for the even pacing in both handbike and wheelchair cycling could be the 259 previous experience of the athlete. On the one hand, he has many years of training experience with 260 many kilometers on the hand bike and, on the other hand, he has already competed in many long 261 competitions with the hand bike. A study investigating predictor variables for a successful finish in a 262 Deca Iron ultra-triathlon found that both the number of finished Triple Iron ultra-triathlons (i.e. 11.4 $263 \mathrm{~km}$ swimming, $540 \mathrm{~km}$ cycling and $126.6 \mathrm{~km}$ running) and the personal best time in a Triple Iron 264 ultra-triathlon were related to overall race time in a Deca Iron ultra-triathlon [23].

266 A further interesting finding was a negative correlation between performance and CV in the Deca 267 Iron ultra-triathlon and a positive correlation was between performance and CV in the Quintuple Iron 
268 ultra-triathlon for the handbike. These correlations are most probably due to his breaks for eating

269 leading to the decrease in speed in the middle of the handbike split.

270

271 Regarding the race strategy of the athlete he always organized his meals according to the same

272 scheme during an Ironman with a first break for eating at around 1 p.m. after $90 \mathrm{~km}$ of handbike

273 cycling followed by a second break shortly after and a third break at around 4 p.m. before changing

274 to the wheelchair split. This strategy was most likely the result of his experience in earlier races

275

276 After $90 \mathrm{~km}$ on the handbike, he took a lunch break of $20 \mathrm{~min}$ at around $1 \mathrm{p} . \mathrm{m}$. and ate the first half

277 of lunch offered by the organizer. After eating, he went to the toilet. He then did another lap of

278 handbike, ate then the second half of the lunch and took coffee and cake. At around 4 p.m., he took

279 another full meal offered by the organizer with the intention that the food should be digested by

280 around 5:30 p.m. before the start of the split with the racing wheelchair.

281

282 Most probably he developed this nutrition strategy during his earlier races. Very little is known

283 regarding nutrition in wheelchair athletes [26]. In able-bodied ultra-endurance cyclists, it is known,

284 however, that appropriate nutrition during a race is an important predictor for a successful race

285 outcome [27]. We must also be aware that a wheelchair athlete must use both his hands during

286 handbike and wheelchair cycling and has no possibility to eat and drink while cycling.

287

288 Anthropometric and physiological data across race days, such as body mass, body water, heart rate

289 and fasting glucose would be important variables to monitor and analyze, but unfortunately the

290 athlete and staff crew were unable to record this data during the race. This could be pointed as a

291 limitation and a goal for future studies. 
292 A limitation of the present study was the specific characteristics (e.g. length of race and disciplines)

293 of 5x and 10xIronman considered in the present study; thus, caution would be needed to generalize

294 these findings in other triathlon races. On the other hand, strength was the novelty of the study as it

295 was the first one to analyze the performance characteristics of an Ironman triathlete. In addition,

296 these findings would have practical applications for both scientists (e.g. model muscle fatigue in

297 upper body ultra-endurance exercise compared to full-body exercise) and professionals working with

298 wheelchair athletes (e.g. develop pacing strategies). Although the success of this athlete in these

299 races was achieved without a close follow-up of an exercise specialist or coach, we do not

300 recommend amateur athletes to prepare for such events without professional input. The ability the

301 organize a training regimen to optimize performance and decrease the chances of burnout and

302 injuries requires knowledge and experience.

303

304 Conclusion

305 An experienced paraplegic wheelchair athlete was able to finish both a Quintuple and a Deca Iron

306 ultra-triathlon. The athlete applied an even pacing in all split disciplines and for overall race time and

307 finished both Quintuple and Deca Iron ultra-triathlon within the time limits of the races.

308

309 Conflict of Interest

310 The authors report no conflicts of interest 


\section{References}

314 1. Knechtle B, Käch I, Rosemann T, et al. The effect of sex, age and performance level on 315 pacing of Ironman triathletes. Research in Sports Medicine. 2019;27(1):99-111.

316 2. Gallmann D, Knechtle B, Rüst CA, et al. Elite triathletes in 'Ironman Hawaii' get older but 317 faster. Age. 2014;36(1):407-416.

318 3. Barbosa LP, Sousa CV, Sales MM, et al. Celebrating 40 years of ironman: How the 319 champions perform. International Journal of Environmental Research and Public Health. $320 \quad 2019 ; 16(6)$.

321 4. Lepers R, Rüst CA, Stapley PJ, et al. Relative improvements in endurance performance with 322 age: Evidence from 25 years of Hawaii Ironman racing. Age. 2013;35(3):953-962.

323 5. Abbiss CR, Laursen PB. Describing and understanding pacing strategies during athletic $324 \quad$ competition. Sports Medicine. 2008;38(3):239-252.

3256 Wu SS, Peiffer JJ, Brisswalter J, et al. Factors influencing pacing in triathlon. Open Access J $326 \quad$ Sports Med. 2014;5:223-34.

327 7. Angehrn N, Rüst CA, Nikolaidis PT, et al. Positive pacing in elite ironman triathletes. 328 Chinese Journal of Physiology. 2016;59(6):305-314.

329 8. Johnson EC, Pryor JL, Casa DJ, et al. Bike and run pacing on downhill segments predict 330 Ironman triathlon relative success. Journal of Science and Medicine in Sport. 2015;18(1):8233187

332 9. Abbiss CR, Quod MJ, Martin DT, et al. Dynamic pacing strategies during the cycle phase of 333 an ironman triathlon. Medicine and Science in Sports and Exercise. 2006;38(4):726-734.

334 10. Abbiss CR, Laursen PB. Describing and understanding pacing strategies during athletic 335 competition. Sports Med. 2008;38(3):239-52.

336 11. Foster C, deKoning JJ, Hettinga F, et al. Effect of competitive distance on energy expenditure 337 during simulated competition. Int J Sports Med. 2004 Apr;25(3):198-204. 
338 12. Sousa CV, Nikolaidis PT, Knechtle B. Ultra-triathlon - pacing, performance trends, the role of nationality and sex differences in finishers and non-finishers. Scand J Med Sci Sports. 2019 Nov 12.

341 13. Noakes TD. Time to move beyond a brainless exercise physiology: the evidence for complex regulation of human exercise performance. Applied physiology, nutrition, and metabolism = Physiologie appliquee, nutrition et metabolisme. 2011 Feb;36(1):23-35.

14. Atkinson G, Brunskill A. Pacing strategies during a cycling time trial with simulated headwinds and tailwinds. Ergonomics. 2000 Oct;43(10):1449-60.

15. Swain DP. A model for optimizing cycling performance by varying power on hills and in wind. Med Sci Sports Exerc. 1997 Aug;29(8):1104-8.

16. Sousa CV, Pereira RW, Rosemann T, et al. Self-Selected Pacing During a World Record Attempt in 40 Ironman-Distance Triathlons in 40 Days. Int J Environ Res Public Health $2020 ; 17(7)$

351 17. Hollander K, Kluge S, Glöer F, et al. Epidemiology of injuries during the Wheelchair 352 Basketball World Championships 2018: A prospective cohort study. Scandinavian Journal of Medicine and Science in Sports. 2019.

354 18. Haydon DS, Pinder RA, Grimshaw PN, et al. Wheelchair Rugby chair configurations: an 355 individual, Robust design approach. Sports Biomechanics. 2019.

19. Schipman J, Gallo P, Marc A, et al. Age-related changes in para and wheelchair racing athlete's performances. Frontiers in Physiology. 2019;10(MAR).

358 20. Teutsch U, Knechtle B, Rüst CA, et al. Age group athletes in inline skating: Decrease in 359 overall and increase in master athlete participation in the longest inline skating race in Europe - The inline One-Eleven. International Journal of General Medicine. 2013;6:345-355.

361 21. Vernillo G, Temesi J, Martin M, et al. Mechanisms of Fatigue and Recovery in Upper versus Lower Limbs in Men. Med Sci Sports Exerc. 2018 Feb;50(2):334-343. 
363 22. Knechtle B, Rosemann T, Lepers R, et al. A comparison of performance of Deca Iron and 364 Triple Deca Iron ultra-triathletes. SpringerPlus. 2014;3(1).

365 23. Herbst L, Knechtle B, Lopez CL, et al. Pacing strategy and change in body composition 366 during a Deca iron triathlon. Chinese Journal of Physiology. 2011;54(4).

367 24. Birkenstock Bicycles. 2018; [cited 2020 April 5]. Available from: https://speedbikes.ch

368 25. Union Cycliste Internationale. Paracyclisme [cited 2020 April 5]. Available from:

$369 \quad$ https://www.uci.org/para-cycling/classification

370 26. Sanz-Quinto S, Moya-Ramón M, Brizuela G, et al. Nutritional strategies in an elite

371 wheelchair marathoner at 3900 m altitude: A case report. Journal of the International Society 372 of Sports Nutrition. 2019;16(1).

373 27. Knechtle B, Knechtle P, Rust CA, et al. Finishers and nonfinishers in the 'Swiss Cycling 374 Marathon ' to qualify for the 'Race Across America '. Journal of strength and conditioning 375 research. $2011 \mathrm{Dec} ; 25(12): 3257-63$.

376 
377 Table 1. Race time in the Deca Iron ultra-triathlon

\begin{tabular}{lcccc}
\hline & \multicolumn{3}{c}{ Race time (hours:minutes: seconds) } \\
\cline { 2 - 5 } & Overall & Swimming & Handbike & Wheelchair \\
\hline Day 1 & $13: 36: 19$ & $01: 41: 30$ & $08: 48: 59$ & $02: 51: 18$ \\
Day 2 & $13: 48: 32$ & $01: 48: 15$ & $08: 42: 37$ & $03: 00: 06$ \\
Day 3 & $13: 56: 11$ & $01: 56: 46$ & $08: 38: 40$ & $03: 11: 16$ \\
Day 4 & $13: 45: 38$ & $01: 53: 15$ & $08: 49: 43$ & $02: 52: 55$ \\
Day 5 & $13: 31: 40$ & $01: 52: 20$ & $08: 30: 28$ & $02: 58: 48$ \\
Day 6 & $13: 29: 12$ & $01: 54: 40$ & $08: 22: 11$ & $03: 02: 10$ \\
Day 7 & $14: 01: 13$ & $01: 54: 21$ & $08: 28: 57$ & $03: 29: 02$ \\
Day 8 & $14: 08: 44$ & $01: 54: 03$ & $08: 55: 30$ & $03: 10: 06$ \\
Day 9 & $13: 40: 01$ & $01: 52: 06$ & $08: 38: 58$ & $02: 54: 05$ \\
Day 10 & $13: 05: 50$ & $01: 48: 04$ & $08: 11: 23$ & $02: 54: 51$ \\
Average & $13: 42: 20$ & $01: 51: 32$ & $08: 36: 45$ & $03: 02: 28$ \\
(SD) & $(18: 07)$ & $(04: 27)$ & $(13: 37)$ & $(11: 34)$ \\
Total & $137: 03: 20$ & $18: 35: 20$ & $86: 07: 26$ & $30: 24: 37$ \\
\hline
\end{tabular}

378 SD: standard deviation (minutes: seconds). Each day consists of $3.8 \mathrm{~km}$ swimming, $180 \mathrm{~km}$ of 379 handbike (cycling split) and $42 \mathrm{~km}$ of wheelchair cycling (running split). 
381 Table 2. Race time in the Quintuple Iron ultra-triathlon

\begin{tabular}{lllll}
\hline \multicolumn{5}{l}{ Race time (hours:minutes:seconds) } \\
\cline { 2 - 5 } & Overall & Swimming & Handbike & Wheelchair \\
\hline Day 1 & $13: 21: 59$ & $01: 41: 38$ & $08: 23: 17$ & $03: 07: 44$ \\
Day 2 & $12: 57: 37$ & $01: 42: 55$ & $08: 22: 28$ & $02: 42: 14$ \\
Day 3 & $13: 10: 55$ & $01: 42: 23$ & $08: 33: 48$ & $02: 43: 31$ \\
Day 4 & $13: 24: 11$ & $01: 45: 51$ & $08: 32: 48$ & $02: 55: 54$ \\
Day 5 & $13: 33: 49$ & $01: 44: 05$ & $08: 38: 57$ & $02: 57: 10$ \\
Average & $13: 17: 42$ & $01: 43: 22$ & $08: 30: 16$ & $02: 53: 19$ \\
(SD) & $(12: 24)$ & $(01: 28)$ & $(06: 23)$ & $(09: 28)$ \\
Total & $66: 28: 31$ & $8: 36: 52$ & $42: 31: 18$ & $14: 26: 33$ \\
\hline
\end{tabular}

382 SD: standard deviation (minutes: seconds). Each day consists of $3.8 \mathrm{~km}$ swimming, 180

$383 \mathrm{~km}$ of handbike (cycling split) and $42 \mathrm{~km}$ of wheelchair cycling (running split). 


\section{$384 \quad$ Figure captions}

385

386 Figure 1. Pacing in each of the 10 days in a Deca Iron ultra-triathlon in the handbike

387 and wheelchair of a Paralympic triathlete; HM: half-marathon; M: marathon.

388

389 Figure 2. Pacing variation and average speed in each of the 10 days in a Deca Iron

390 ultra-triathlon in the handbike and wheelchair of a Paralympic triathlete.

391

392 Figure 3. Pacing in each of the 5 days in a Quintuple Iron ultra-triathlon in the handbike and wheelchair of a Paralympic triathlete; HM: half-marathon; M: marathon.

394

395 Figure 4. Pacing variation and average speed in each of the 5 days in a Quintuple Iron

396 ultra-triathlon in the handbike and wheelchair of a Paralympic triathlete.

397

398 Figure 5. Correlation analysis between pacing variation and average performance in a 399 Deca and a Quintuple Iron ultra-triathlon in the handbike and wheelchair of a 


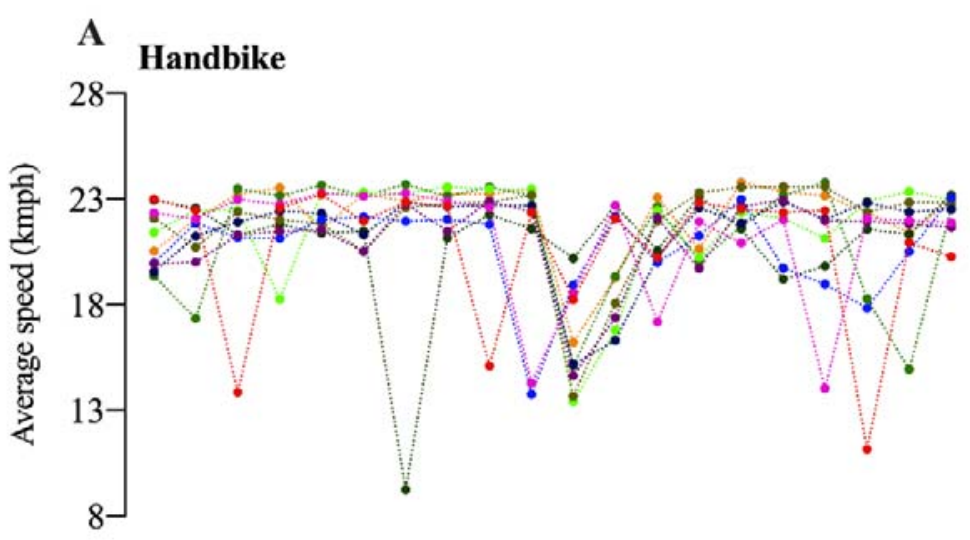

-... Day 1

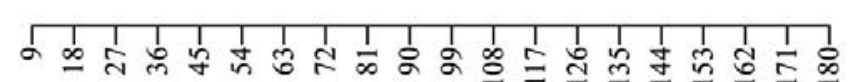

•... Day 2

Splits (Km)

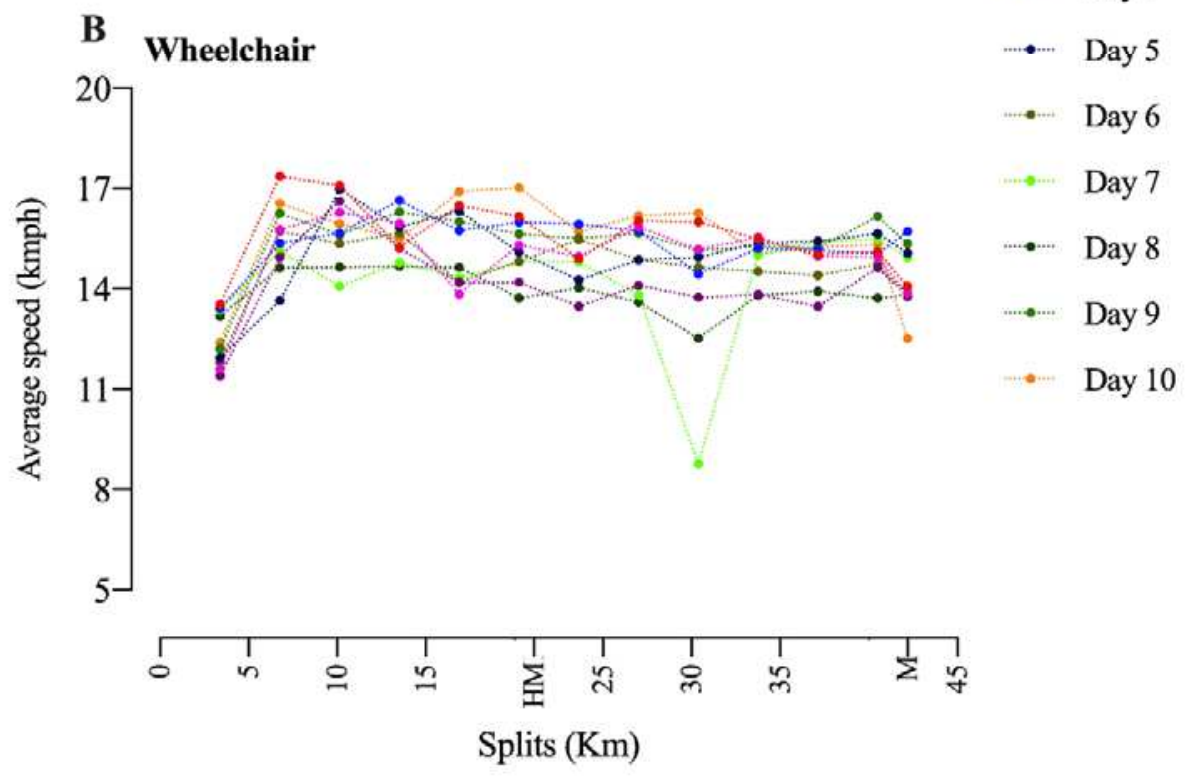

401

e.

402

403 

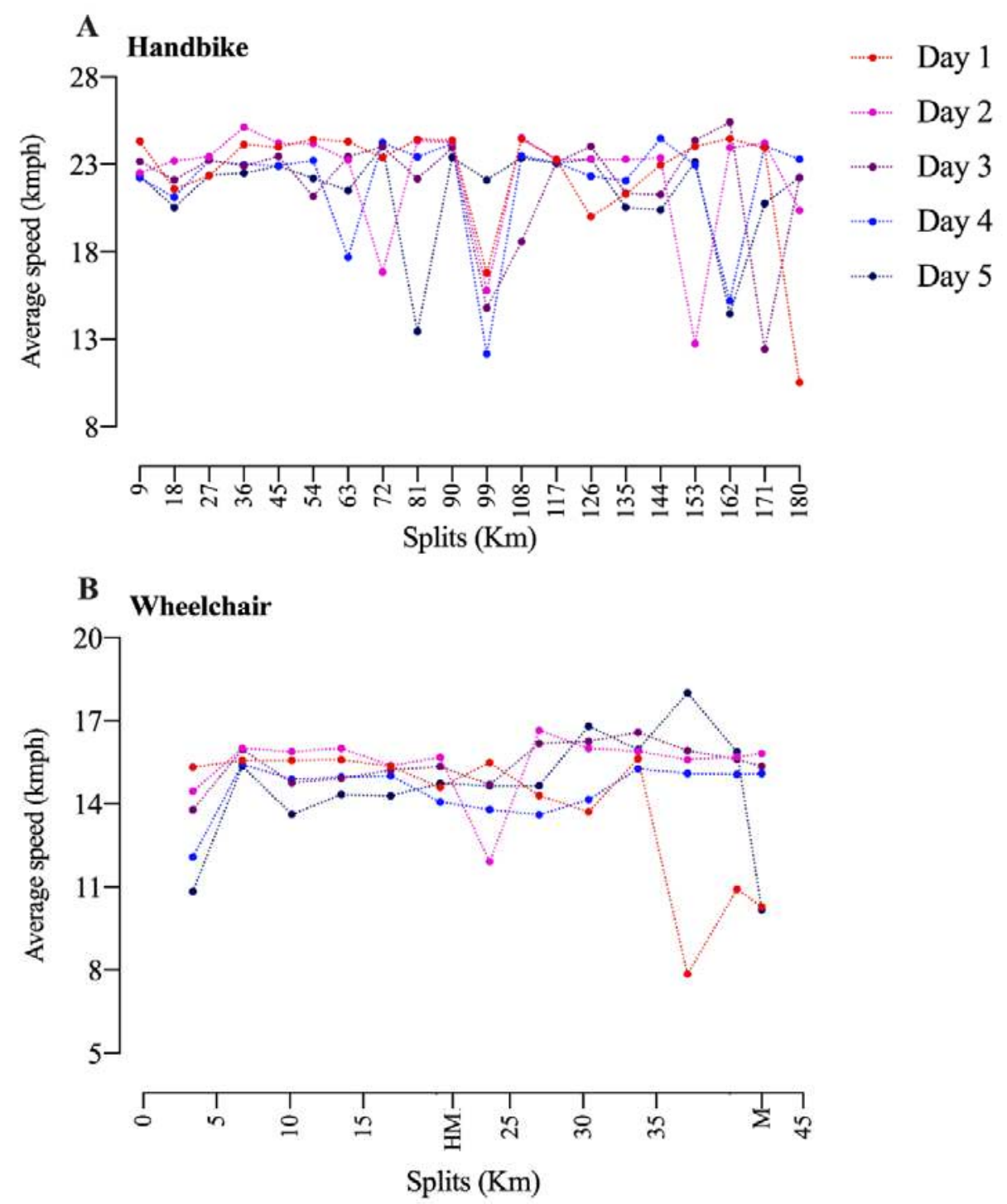

404

A
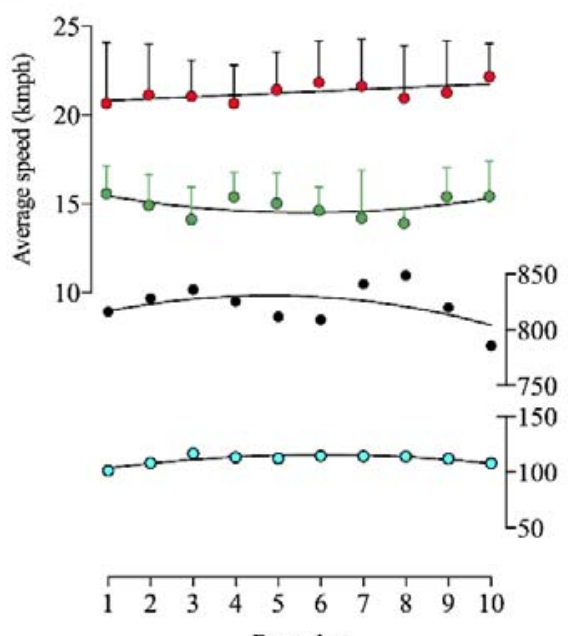

405
B

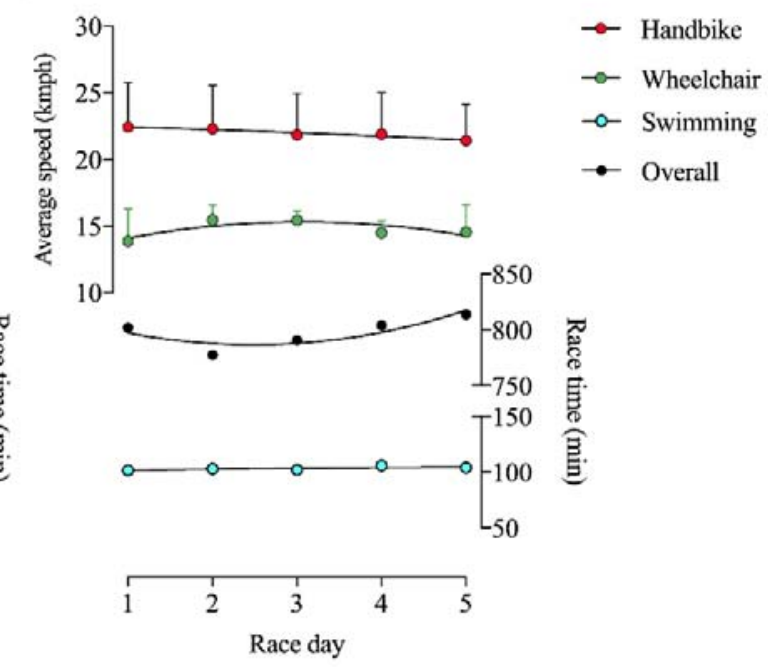


A

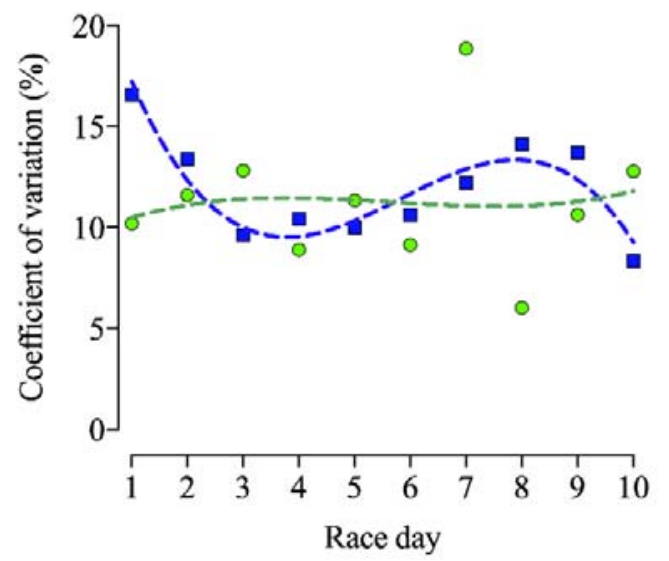

B

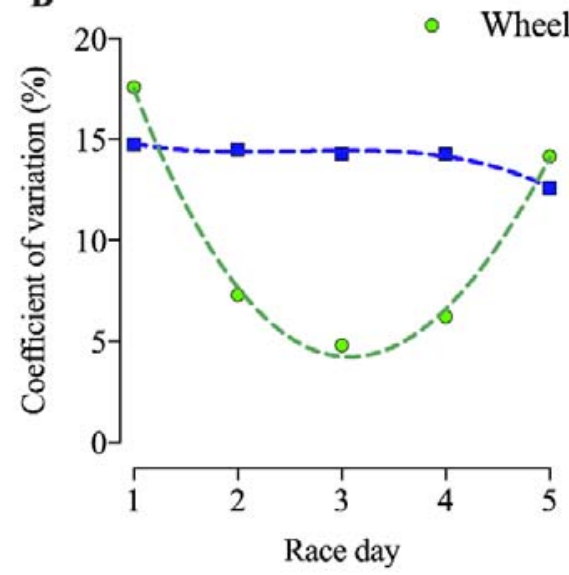

407

\section{Deca Iron Ultra Triathlon}

Handbike

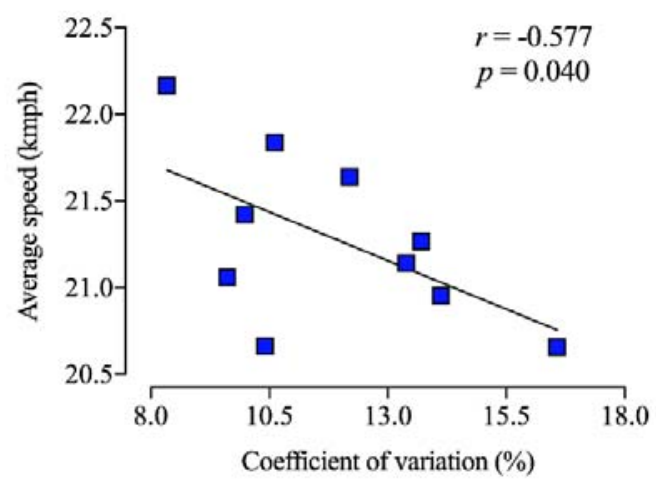

Wheelchair

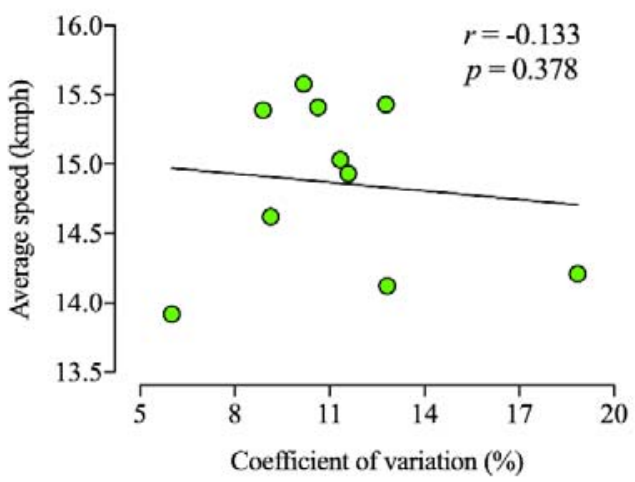

\section{Quintuple Iron Ultra Triathlon}

Handbike

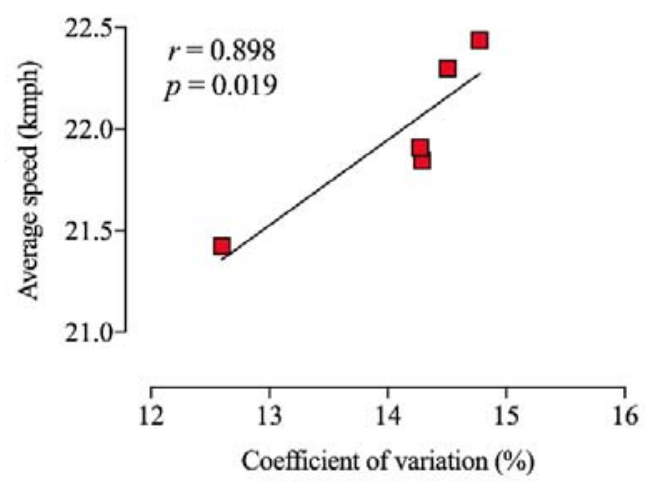

Wheelchair

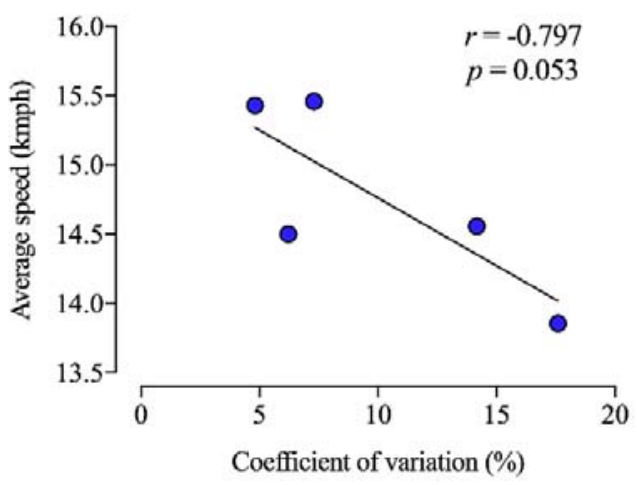

